\title{
Empirically analysing design reasoning patterns: Abductive-deductive reasoning patterns dominate design idea generation
}

Claus L. Cramer-Petersen, Department of Management Engineering,

Technical University of Denmark, DK-2800 Kongens Lyngby, Denmark

Bo T. Christensen, Department of Marketing, Copenhagen Business School, DK-2000 Frederiksberg, Denmark

Saeema Ahmed-Kristensen, School of Design, Royal College of Art, London SW7 2EU, UK

Reasoning is a fundamental process in design activity, and it provides a way to understand design behaviour. Theories and models of design propose reasoning that follows abductive-deductive patterns. At the micro-level, these patterns are untested. This study analyses verbal reasoning patterns at the micro-level for group idea generation using protocol analyses of concurrent verbalisations from five design teams with industry participants. The results show that reasoning in design activity across 218 ideas follows general patterns of abductive-deductive reasoning. At the individual idea level, the reasoning patterns are disorderly and enter into micro-patterns of inference. The study concludes that understanding reasoning at early-stage idea generation processes is indicative of the mental models and abductive-deductive reasoning that are prevalent in design activity. (c) 2018 Elsevier Ltd. All rights reserved.

Keywords: design activity, design reasoning, protocol analysis, logic of design, idea generation

Corresponding author: C. L. Cramer-Petersen claus.lcp@gmail.com

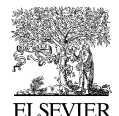

$\mathrm{R}$ easoning is at the heart of design activity, and it determines how humans respond to situations in every aspect of their lives (JohnsonLaird, 2009). The reasoning of designers consists of trains of thought, including deliberation, argumentation and making logical inferences (Rittel, 1987), and it is central to understanding and supporting design activity.

Drawing on the works of Peirce (1980), inferences in design are described as being driven by abductive reasoning (Dorst, 2011; Roozenburg, 1993), meaning that design activity begins by hypothesising desired outcomes or functions, and it moves towards proposing forms and structures that can realise such desired outcomes. Pertaining to the processes involved in design, different models describe the activity as comprising stages that begin by formulating

www.elsevier.com/locate/destud 0142-694X Design Studies 60 (2019) 39-70

https://doi.org/10.1016/j.destud.2018.10.001

(c) 2018 Elsevier Ltd. All rights reserved. 
initial hypotheses to propose desired functions followed by the generation of probable behaviours and solutions to such functions, involving evaluation and reflection on which solutions are suitable for the desired function (March, 1976; Schön, 1991). Such processes involve both abductive, deductive and inductive reasoning; they are learning processes that do not follow strict abductive-deductive-inductive sequences (March, 1976).

Rittel (1987) describes design activity as a disorderly process that is inherently argumentative in its reasoning. Design activity involves the negotiation among the different perspectives and desires of those involved in a design process (Bucciarelli, 2002). Hence, design is understood as a social process through which solutions emerge as the result of argumentation among the different perspectives and values of those involved in the process. In such processes, reasoning is reflected by the deliberation and arguing among designers where language plays a performative role in that it enacts and constitutes design (Dong, 2007).

However, the related empirical studies of the reasoning and structure of design activity have in common that they concern design activity in relation to the proposition, detailing and evaluation of objects through inference by applying 'design thinking'. Such analyses are either performed conceptually (Dorst, 2011; March, 1976; Roozenburg, 1993) or they rely on verbal reports that (post)rationalise the activity undertaken (e.g., Galle, 1996b; Lloyd \& Scott, 1994), making them unsuitable for capturing the argumentative aspects of reasoning processes as they occur in situations that are contentious and influenced by diverging values, objectives and desires (Bucciarelli, 2002; Stumpf \& McDonnell, 2002).

Consequently, the study sets out to empirically analyse reasoning in a verbal form as it occurs among people engaged in design activity. This necessitates combining a tradition for describing a logic of design through formal reasoning (March, 1976; Roozenburg, 1993) with notions of design as enacted through language (Dong, 2007) among individuals with differing intentions and perceptions (Bucciarelli, 2002). The study aims to contribute with a novel analysis and perspective on empirical reasoning in design and its implications for design in practice.

\section{Theory and background}

\subsection{Logical reasoning}

Since the works of C.S. Peirce in the mid-20th century and earlier, logical reasoning has been formulated as being deductive, inductive or abductive (Peirce, 1980). These reasoning types define three distinctly different ways of drawing conclusions from premises. 
Deductive reasoning is self-referencing as it allows for the reaching of a conclusion based on the logical implications of two or more propositions asserted to be true (Magnani, 1995; March, 1976; Reichertz, 2010). Consequently, deduction is justificational as the premises guarantee the truth of a conclusion (Schurz, 2007). An example of deductive reasoning is as follows:

The weather is sunny. John only brings his umbrella when it rains, so he will not bring it today.

Inductive reasoning is the process of deriving plausible conclusions that go beyond the information in the premises (Johnson-Laird, 2009). Inductive reasoning is self-referencing in a manner that is similar to deductive reasoning in that it infers concepts only from available data within a model or frame of reference (Magnani, 1995; Reichertz, 2010; Schurz, 2007). Unlike deduction, induction does not produce guaranteed true conclusions as deduction does. Instead, inductive reasoning infers conclusions that go beyond the available data. An example of inductive reasoning is as follows:

Upon having drawn five white marbles from the bag, Peter concludes that all the remaining marbles in the bag must be white.

Abductive reasoning is a process of conjecture that yields the best (and simplest) explanation to a course of events. An abduction is the preliminary estimate that introduces plausible hypotheses and informs where to first enquire by choosing the best candidate among a multitude of possible explanations (Magnani, 1995; Schurz, 2007). Therefore, abduction is reductive; however, it does not, unlike induction, require one to draw conclusions from available data. Abductive reasoning differs from deductive and inductive reasoning in that abduction involves guessing and (sometimes unfounded) assumptions as the basis for reasoning. An example of abductive reasoning is as follows:

Lisa's fingerprints were on the gun that shot Michael. Lisa is suspected of firing the gun.

In design activity, abductive reasoning involves the conception of new rules or types of relationships to explain an intended outcome (Roozenburg, 1993). This innovative potential of abductive reasoning makes it a creative feat, by definition, and thus also necessary in the generation of anything original (Dorst, 2011; Reichertz, 2010). In contrast, neither deductive nor inductive reasoning can propose entirely new ideas or concepts because they depend on available data to draw conclusions (Peirce, 1980).

The logic of reasoning in design is proposed to be an abductive activity moving from function to form (Dorst, 2011; Roozenburg, 1993). In cases of reasoning 
leading to innovation or new ideas, such abductions are termed as innoduction (Roozenburg, 1995) or abduction-2 (Dorst, 2011) signifying a type of reasoning that moves from an aspired value or function towards a form, but without knowing either the working principle or form beforehand. A more recent proposal elaborates this process as a two-step abductive process that invents a form (design object), a mode of use and a mode of action to fulfil the desired function (Kroll \& Koskela, 2015). One way to exemplify reasoning in design used originally by Roozenburg (1993) and later elaborated by Kroll \& Koskela (2015) is the imagined first development of a kettle to boil water using a stove. The first abduction pertains to the desired outcome of making water boil (function), which necessitates placing water on the burner for heating, concluding one, among many possible, modes of action. The second abduction concludes that a device to hold the water in place (form) is one, among many possible, viable solutions on the premise of the just-concluded modes of action. Thus, abduction is also termed as the inference to the best explanation because it involves a particularly promising conjecture (Roozenburg, 1993; Schurz, 2007).

In the above definitions and examples, reasoning processes are conceptual and assume a logically sound reasoning pattern whereby abductive reasoning is the only reasoning type with the potential to create something new (Dorst, 2011; Roozenburg, 1993).

\subsection{Premises for reasoning}

Per the definition, the reasoning types of deduction, induction and abduction define ways of making inferences from premises that reflect the knowledge and beliefs held by the person engaged in reasoning (Dorst, 2011). Such knowledge and beliefs form - and stem from - mental models (Johnson-Laird, 1983). Mental models are constructs that organise knowledge pertaining to specific contexts (Klimoski \& Mohammed, 1994) and are thus relevant in the understanding of human behaviour and reasoning in design activity (Badkeschaub, Lauche, Neumann, \& Ahmed, 2007). Mental models are not fixed, and they change according to the new experiences and knowledge created in relevant situations (Johnson-Laird, 2009). Thus, as logical reasoning is based on mental models (Johnson-Laird, 1983), inferences drawn from reasoning are not fixed and vary among individuals. Hence, empirically studying reasoning implies that the reasoning observed reflects, and depends on, the individual mental models held.

Rittel (1987) defines reasoning in design as a process of argumentation. In design activity, this results in issues and competing positions that are simultaneously interconnected and 'open' as a consequence of different mental models. When engaged in a verbal discourse, these divergent perspectives can appear as speculation, argumentation, trade-offs or negotiation 
(Bucciarelli, 2002; Rittel, 1987). Furthermore, when reasoning in a context of dialogue with other people, a person uses mental models, implicitly or explicitly, to create a frame of understanding, which in turn allows for the generation and description of solutions-design activity. As design is a social process (Bucciarelli, 2002) involving the differing perspectives of those involved, ideas are not necessarily understood or accepted by the audience, resulting in an argumentative interaction among the participants (Stumpf \& McDonnell, 2002). Thus, the 'logic' of verbal reasoning takes the form of conclusions based on premises that both draw on existing understandings (facts) and on values (Stumpf \& McDonnell, 2002) and thus diverge from the logical definitions of reasoning that assume a universal truth for deductive reasoning and a strict adherence to only what is observed for inductive reasoning (Peirce, 1980).

Taking the definition of reasoning in design as a process of argumentation at face value (Rittel, 1987), the field of argumentation theory and rhetoric offers insights to explain reasoning in groups of people. Argumentation theory defines argumentation as an integral part of reasoning (Mercier \& Sperber, 2011). Thus, analysing conversation in groups of people engaged in design holds the potential to facilitate an understanding of and explain verbal reasoning as the deployment of linguistic processes to satisfy the demands of reasoning (Polk \& Newell, 1995). Such attempts at verbal reasoning derive their effectiveness from their similarity to formal types of reasoning (Perelman \& Olbrechts-Tyteca, 1973). A consonant explanation from the field of cognitive science is that the beliefs and knowledge held in the mental models used to reason about specific tasks or events are held in working memory and may therefore be articulated (Christensen \& Schunn, 2009).

Verbal reasoning is therefore not identical to the deductive, inductive or abductive reasoning types in the formal logical sense; however, a verbal realisation bears similarities to logical reasoning types in their verbal deployment. Therefore, perceiving group dialogues as a process of argumentation is representative of the underlying reasoning with the important implications that a) the reasoning reflects the deployment of a mental model that might be different from the ones held by those addressed in verbal reasoning (Badke-schaub et al., 2007; Johnson-Laird, 1983) and that b) the verbal enactment of such reasoning is influenced by values and beliefs and in turn acts to propose a certain perspective (Dong, 2007; Stumpf \& McDonnell, 2002).

\subsection{Models of design activity}

Various models and frameworks emphasise design as a process involving different types of activity and as a process of inquiry. The following section reviews such models of design activity focusing on descriptions or prescriptions of the micro-level steps and thinking involved in design activity, i.e., the different reasoning implied by the models. 
Drawing on the works of Peirce and thus drawing on the formal reasoning types directly, March (1976) proposes the production-deduction-induction (PDI) model. The PDI model proposes a rational design process of cyclic iterative procedures characterised by three different types of reasoning, proposing a productive-deductive-inductive cycle as a necessary element of reasoning in design. First, productive reasoning composes something novel. It suggests that something may be. Second, deductive reasoning decomposes and predicts the performance characteristics of a design that emerge from analysis of the composition. It proves that something must be. Third, inductive reasoning supposes from the accumulation of knowledge and the establishment of values evolving from the prior productive and deductive reasoning. It tests whether something actually is. Empirically testing the PDI model, a study by Lloyd and Scott (1994) analysed the think-aloud protocols of engineering designers for generative, deductive and evaluative reasoning and concluded that reasoning types interact and that generative-deductive-inductive sequences occur during design activity; however, they also found other sequences of reasoning. More recently, the PDI model has been criticised for proposing design to explicitly concern generalisation through inductive reasoning (Koskela, Paavola, \& Kroll, 2018) and to draw overtly from the abductive, deductive, inductive reasoning cycles used in scientific discovery (Magnani, 2004). Hence, there is reason to question the validity of inductive reasoning to have a specific role in design reasoning.

In a study, Galle (1996a) empirically analysed design reasoning through the use of replication protocol analysis by asking an expert architect to replicate the interpreted reasoning underlying other people's work. Using such analysis, he found patterns of inference corresponding to abductive and deductive reasoning and argues that deductive reasoning can be productive and introduce new elements to a design. He further observed that design reasoning is occasionally opportunistic and based on beliefs and therefore does not necessarily reach a strict, formal, logical conclusion as per the premises acting as the basis for reasoning.

In the theory of reflective practice, Schön (1991) proposes a perspective on design activity. Acknowledging that design contains logical design patterns consisting of 'if... then' propositions that occur in cumulative sequences from prior decisions, Schön emphasises the different contextual norms drawn by the domains of different stakeholders involved in design. The process of design, in Schön's perspective, is a practice involving naming, framing, moving and reflecting in cycles converging towards problem understanding and moving towards a solution (Schön, 1991). First, naming focuses explicitly on a part of the design task. Second, framing guides subsequent activity by providing a way for individuals and teams alike to 'see' and shape the design problem. Third, moving generates solutions to solve the problem set by the frame. Fourth, reflecting evaluates moves relating to their desirability. A study by 
Valkenburg and Dorst (1998) using protocol analysis found that a student design team managing both framing and moving activity in integration led to good team performance, thus indicating a dynamic between activities that diverge and allowing new ways to 'see' in combination with the generation of (tentative) solutions to solve, or test, a process similar to mental simulation (Christensen \& Schunn, 2009).

Using the concept of generative sensing, Dong, Garbuio, and Lovallo (2016) describes design reasoning as a pattern of deductive and abductive reasoning that provides different 'ways through the problem' (ibid.: p 3) in the case of design concept evaluation. Aside from finding abductive reasoning present in evaluation, they also argue for abductive reasoning as being directed towards both convergent and divergent thinking, i.e., proposing both new frames of understanding as ways of reaching conclusions. Dong, Lovallo, and Mounarath (2015) also analysed the verbal protocols of reasoning processes among people discussing and evaluating design ideas and concepts in terms of the deductive, inductive and abductive reasoning types, showing that reasoning can be reliably identified from protocols including recorded dialogue in groups of people. They found that the type of reasoning dominant when evaluating ideas influenced the evaluation of the ideas. Abductive reasoning for evaluating tended to result in more ideas being accepted, while the opposite was true for deductive reasoning used for evaluation. Consequently, they argue for further debating and analysing empirical reasoning, as opposed to theoretical observations on reasoning in design from a logical perspective.

An alternative model of design is proposed by Hatchuel and Weil (2008). Concept-knowledge $(\mathrm{C}-\mathrm{K})$ emphasises the interplay between what is conjectured or unknown and what is known or in existence, and it describe the operators between concept and knowledge. One such operator is between knowledge and thus consist of logical reasoning (akin to that proposed by Peirce). Other operators allow for the making of concepts and assessing them through available (accepted) knowledge or, conversely, using knowledge to inform and generate new concepts. Hence, $\mathrm{C}-\mathrm{K}$ theory suggests a dynamic between ways of thinking that are tentative and that which is accepted, factual or otherwise taken for granted.

\subsection{Patterns of reasoning in design}

The reviewed models and studies of design activity all describe the activity as going through stages that enter into iterations or re-formulations towards a solution. In common for all models is the notion of sequences of activity that iterate between activities that propose a new perspective on a design task, regardless of whether they are termed compositions, frames, or ways to perceive on the one hand, and activities that seek to describe, predict or 
move towards design solutions on the other hand. While some of the reviewed models also explicitly state the existence of an activity concerning the evaluation and reflection of the previous, this activity seems more doubtful. Thus, the approaches have in common that they describe design activity as being iterative, involving cycles of reasoning towards solutions and being a process of learning about the problem through the generation of solutions, resulting in the co-evolution of problem and solution (Dorst \& Cross, 2001).

Despite the relative agreement in the models that design activity iterates between hypothesis generation and exploration, there are results showing that the underlying reasoning types in some cases function in discordance with their strictly formal definitions. An example of this is the arguments by Galle (1996a) that deductive reasoning does in some cases produce new solutions, or that abductive reasoning is also prevalent in the evaluation of design concepts (Dong et al., 2016). Pertaining to design activity, Rittel (1987) argues that there is no clear separation between problem definition, synthesis and evaluation in real-world design activity and that 'only at the micro-level can we identify patterns of reasoning corresponding to [the design process]' (Rittel, 1987, p. 3).

\subsection{Analysing micro-level reasoning in design}

As shown in the above review of the existing research on reasoning in design, little or no research has been done on the reasoning patterns present at the micro-level of design activity comprising the inference made at the level of individual arguments between groups of designers. Research is therefore desired to identify and understand reasoning patterns within arguments - what this study defines as micro-level design activity (Dong et al., 2015; Rittel, 1987). The decision to analyse micro-level reasoning implies parting with the thusfar established perception of reasoning as containing premises, rules and conclusions. The present approach allows for analysing entire reasoning patterns whereas the previous studies determine a single type of reasoning. Arguably, this allows for a more fine-grained understanding of how design activity takes place.

Synthesising the above review, the paper proposes to empirically test whether the characteristics of design activity show a pattern if interpreted as a reasoning process. Departing from the above-described formal definitions of deductive, inductive and abductive reasoning as distinct types and combining that with the reviewed models of design activity, a process of reasoning in design would involve 1) abductive reasoning that leads to a problem setting by framing and suggesting functions, followed by 2) deductive reasoning that concretises the solution and predicts its effects on the problem set. Finally, a more debated phase might involve inductive reasoning that evaluates design activity. The two-stage process is similar to a definition from the field of 
cognitive science that describes a generic problem-solving cycle as a multistage process involving the '...use [of] some constraints to generate a putative solution, and other constraints, such as the goal of the problem, to criticise and amend the results' (Johnson-Laird, 2009).

Based on results from above reviewed studies of reasoning in design, design activity, and consequently design reasoning, is not expected to follow the two-stage process in a strict and homogeneous way. Nonetheless, the commonalities explained above across models of design activity invites investigation into the degree to which such a pattern of abductive-deductive reasoning does indeed exist and what the role of inductive reasoning might be.

\subsection{Idea generation as an instance of design activity}

A specific stage of the design process is the generation of design ideas whereby new ideas are put forward and explored. Dictionaries commonly define ideas as concerning 1) an imagined outcome, 2) a course of action and 3) the basis on which something is believed to be valid (Merriam-Webster; Oxford). Hence, perceiving ideas as a process of inference is consonant with the above descriptions of the reasoning processes underpinning design activity because the notion of an idea contains a setting and/or proposition for an imagined outcome as well as a description towards an actual solution. The early stage of idea generation provides a way to investigate the proposed abductivedeductive patterns within short time intervals, allowing for a higher number of episodes suitable for analysing patterns in reasoning.

Furthermore, idea generation designates a key part of the design process (Cross, 2001) that regards the formulation of, and trade-offs between, issues of key design features (Ahmed, Wallace, \& Blessing, 2003; Bucciarelli, 2002). Hence, design idea generation is deemed a suitable unit of analysis to investigate reasoning behaviour notwithstanding the potential representativity to design activity in general.

Finally, to arrive at a practical approach to analysing the three-stage reasoning process in a context of idea generation, a simplified separation of ideas into three equal parts is done. This will allow for the analysis of specific concentrations of reasoning types at different stages of design activity. The specific division into three parts is grounded in the prevalence of models of design activity that concern two or more stages of design. While the greatest agreement among these is that of abductive-deductive patterns, as discussed above, there is some disagreement as to the role of evaluation in design activity. Hence, a three-part division of ideas will allow for a greater resolution for analysing reasoning occurrences as ideas develop and also leave room for investigating whether any unexpected patterns of reasoning occur during the end of ideas. 


\section{Aims and hypotheses}

The study aims to understand empirical design reasoning in a context of group design activity in the context of idea generation. The aim is pursued in two ways: first, by testing a set of hypotheses regarding a proposed abductivedeductive pattern with regard to the verbal deployment of reasoning among groups of designers. Second, the study aims to show and discuss how reasoning occurs at the micro-level of design activity. This is done through the use of examples to illustrate the reasoning patterns identified in group idea generation.

Pertaining to the first aim, a set of two hypotheses predict a distribution of reasoning in idea generation resulting from the analysis of reasoning in design and models of design activity.

H1: Abductive (compared to deductive) reasoning is relatively concentrated in the first part of the verbal realisation of an idea.

$\mathrm{H}$ 2: Deductive (compared to abductive) reasoning is relatively concentrated in the middle part of the verbal realisation of an idea.

Finally, a more tentative and exploratory question is posed as to the role of inductive reasoning. While not expected to be prominent in idea generation as it is evaluative (de Bono, 1992), there is an expectation that any inductive reasoning present will be more pronounced in the last parts of generated ideas.

The hypotheses concern an analysis of the proportional distribution of reasoning within design ideas as a unit of analysis, divided in three parts. A first part of abductive reasoning that serves to state an intention or desired result by conjecturing that a specific aspect of the design task is relevant. Next, there is a middle part of deductive reasoning, indicating the concretisation of solutions framed by the initial hypothesis. Finally, the last part of reasoning is more uncertain, in some cases described as being evaluative through induction (March, 1976) Because this model is contested (Koskela et al., 2018), we refrain from making definitive hypotheses about what type of reasoning is more prevalent in the last parts of ideas; however, we venture an exploratory question as to the role of inductive reasoning. Together, the hypotheses predict that idea generation design reasoning follows an abductivedeductive pattern through the ideas generated. As argued above, ideas represent an instantiation of design activity, thus allowing the use of individual idea episodes as a basis for determining reasoning patterns that go through the proposed two-stage process within a limited timeframe. Rittel (1987) proposes that the reasoning patterns in design activity are disorderly. Thus, the hypotheses do not predict a strict adherence to an abductive-deductive pattern but 
rather predict that each reasoning type is concentrated at specific parts of ideas.

Pertaining to the second aim of the study, a qualitative analysis of the reasoning patterns found in the data is conducted to flesh out the implications of the quantitative analysis obtained by testing the hypotheses.

\section{Method}

The study collected data from five teams of three members working on idea generation for the same design task. Protocol coding of transcribed recordings was used as the basis for analysing the data.

\subsection{Data collection}

An experiment was designed for teams of participants from industry engaged in idea generation to perform a specific design task. The participants volunteered to take part in the experiment as part of an innovation workshop. The participants were from different companies and industry sectors. Nine participants were female, and six were male. Table 1 summarises the details pertaining to the participants.

A study by Ahmed et al. (2003) found differences in reasoning activities between novice and experienced designers, warranting data collection focusing on the design activity of experienced professionals since this is more representative of actual design activity and behaviour than e.g., that of novices or students. Regarding the number of participants in the analysis, the argument is twofold. First, obtaining industry professionals is a barrier in terms of obtaining a high number of participants; however, for the reasons outlined above, we preferred to use students; and second, the data segmentation and qualitative coding method approaches 6000 segments, which would be unmanageable and highly time consuming if it were much larger.

Prior to the idea generation session, an introduction to the design task was provided by the main author. Next, teams were generated at random to form five teams of three participants each.

To make the design task understandable for participants of varying backgrounds, it focused on the generation of ideas for radically reducing water consumption at a local hotel. To match the backgrounds of the participants, the design task emphasised the generation of ideas concerning not only technical solutions but also organisational or behavioural ideas, or combinations thereof. The task was formulated by an industry company with a commercial interest in the subject matter of the task before the experiment to ensure the relevance to real life industrial design practice. No participants of the study were from this company. 
Table 1 Background details of participants

\begin{tabular}{|c|c|c|c|c|c|c|c|}
\hline \multicolumn{5}{|c|}{ Participant information } & \multicolumn{3}{|c|}{ Previous working experience } \\
\hline Participant & Group & Gender & Industry sector & $\begin{array}{l}\text { Years of } \\
\text { experience }\end{array}$ & $\begin{array}{l}\text { Problem } \\
\text { solving }\end{array}$ & $\begin{array}{l}\text { Technical } \\
\text { design }\end{array}$ & $\begin{array}{l}\text { Creative } \\
\text { methods }\end{array}$ \\
\hline 1 & 1 & M & Energy & 27 & $\mathrm{X}$ & $\mathrm{X}$ & $\mathrm{X}$ \\
\hline 2 & 1 & $\mathrm{~F}$ & Higher education & 7 & $\mathrm{X}$ & & $\mathrm{X}$ \\
\hline 3 & 1 & $\mathrm{~F}$ & Telecommunication & 41 & $\mathrm{X}$ & & $\mathrm{X}$ \\
\hline 4 & 2 & M & Logistics & 20 & $\mathrm{X}$ & $\mathrm{X}$ & $\mathrm{X}$ \\
\hline 5 & 2 & $\mathrm{~F}$ & Finance & 19 & $\mathrm{X}$ & & $\mathrm{X}$ \\
\hline 6 & 2 & $\mathrm{~F}$ & Publishing & 25 & $\mathrm{X}$ & & $\mathrm{X}$ \\
\hline 7 & 3 & $\mathrm{~F}$ & Graphical design & 1 & $\mathrm{X}$ & $\mathrm{X}$ & $\mathrm{X}$ \\
\hline 8 & 3 & M & Publishing & 35 & $\mathrm{X}$ & & $\mathrm{X}$ \\
\hline 9 & 3 & M & Logistics & 40 & $\mathrm{X}$ & & $\mathrm{X}$ \\
\hline 10 & 4 & $\mathrm{~F}$ & Higher education & 10 & & & $\mathrm{X}$ \\
\hline 11 & 4 & $\mathrm{~F}$ & Public administration & 17 & $\mathrm{X}$ & $\mathrm{X}$ & $\mathrm{X}$ \\
\hline 12 & 4 & M & Organisation & 15 & $\mathrm{X}$ & & $\mathrm{X}$ \\
\hline 13 & 5 & M & Healthcare & 23 & $\mathrm{X}$ & & $\mathrm{X}$ \\
\hline 14 & 5 & $\mathrm{~F}$ & Pharma & 20 & $\mathrm{X}$ & & $\mathrm{X}$ \\
\hline \multirow[t]{2}{*}{15} & 5 & $\mathrm{~F}$ & Insurance & 14 & $\mathrm{X}$ & & $\mathrm{X}$ \\
\hline & & & & $\begin{array}{l}\text { Average }=20.9 \\
{[S D=11.5]}\end{array}$ & $93 \%$ & $27 \%$ & $100 \%$ \\
\hline
\end{tabular}

The team began with a 10-min period to become familiar with the design task and the idea generation process. The period involved discussions between the facilitators and the participants on practical details as well as informal talk and socialisation in the teams. After this, the teams generated ideas, spending 20 min using the three creative methods: brainstorming, random Images and bio-cards. The idea generation methods were intended to create variation over the course of the idea generation sessions. The participants were provided with paper for taking notes or sketching. Table 2 presents the creative methods used for idea generation.

The five teams underwent the idea generation in parallel, in separate rooms. A graduate student of design engineering facilitated each team, instructing the participants to a) allow individual idea generation but ensure that ideas are presented and discussed as a group; b) build on the ideas generated by others, if relevant; and c) ensure timekeeping. The facilitators were blind to the hypotheses and aims of the study, and they followed a printed protocol to ensure that the teams adhered to the time schedule and activities. In some instances, the facilitator contributed to the discussion to ensure that the teams did not get stuck in generating ideas. Since the facilitators were blind to the purpose of the study, their involvement did not interfere with the natural dialogue occurring in the teams.

For all teams, brainstorming was the first method, after which the ordering of the random images and bio-cards methods were presented in random order to avoid any ordering effects caused by ideas generated using previous methods 
Table 2 Creative methods used by teams to generate ideas

\begin{tabular}{|c|c|c|c|}
\hline Method & $\begin{array}{c}\text { Idea generation with no } \\
\text { instructions }\end{array}$ & Random images & Bio-cards \\
\hline Description & $\begin{array}{l}\text { Teams were instructed to } \\
\text { brainstorm together to } \\
\text { generate ideas, following } \\
\text { the principles of } \\
\text { generating many ideas } \\
\text { and withholding criticism } \\
\text { (Osborn, 1953). }\end{array}$ & $\begin{array}{l}\text { Each team was given six } \\
\text { random images from a } \\
\text { catalogue of } 100 \text { random } \\
\text { images drawn from the } \\
\text { past research on idea } \\
\text { generation in design } \\
\text { (Christensen, 2010). } \\
\text { Images relating to water } \\
\text { or nature were not used in } \\
\text { the pool from which the } \\
\text { images were drawn to } \\
\text { avoid overlap with bio- } \\
\text { cards. }\end{array}$ & $\begin{array}{l}\text { Each team was given six } \\
\text { bio-cards created using } \\
\text { the Ask Nature } \\
\text { Biomimicry Taxonomy } \\
\text { (Ask Nature). Six } \\
\text { functions were picked at } \\
\text { random from taxonomy } \\
\text { functions relating to } \\
\text { water, including functions } \\
\text { relating to filtering, } \\
\text { transporting, collecting } \\
\text { and optimising water } \\
\text { (consumption). }\end{array}$ \\
\hline
\end{tabular}

that included providing the participants with inspirational material. The facilitators began each method with a short introduction, after which the participants began generating ideas.

Video and audio recordings were used to collect the data of the idea generation process, resulting in a total of $5 \mathrm{~h}$ and $36 \mathrm{~min}$ of video of design interaction from the five teams (varying from 62 to 73 min per team).

\subsection{Analysis method}

The data were analysed using protocol analyses of concurrent verbalisation. Protocol analysis of design activity is appropriate to understand underlying cognitive processes such as reasoning, with minimal interruption of the recorded process (Ericsson \& Simon, 1993). Consequently, verbal protocol analyses of practitioners from industry is relevant and expected to be highly representative of design cognition found in practice (Ahmed et al., 2003; Chi, 1997; Christensen \& Ball, 2014). In this case, as the observations were in teams, no forced or primed instructions for the participants to thinkaloud were given, resulting in a minimum of interference with thought processes.

The idea generation sessions were transcribed. To analyse these protocols of verbal data in depth, qualitative coding was considered to be a viable method (Chi, 1997). To prepare for the coding of reasoning at a micro-level, segmentation was completed according to word phrases to allow the individual coding of utterances of the shortest possible meaningful length (Goldschmidt, 2009).

Next, a two-step coding scheme was developed to analyse the segmented protocols. 
The first step of analysing the protocols involved the identification of the ideas uttered by the teams. Table 3 presents the code names and definitions.

As ideas involve solutions and sub-solutions (idea aspects) (Badke-schaub et al., 2007), it is necessary to perceive ideas as being put forward in a distributed manner, and at different levels of abstraction (Voss, 2006). More than one participant can contribute to the generation of ideas. Consequently, the protocols do not distinguish complete uninterrupted utterance sequences but groups of utterances relating to an idea put forward and related aspects of that idea. This group of segments is referred to as an idea episode (Chi, 1997). To test the hypotheses, each idea episode was separated into three parts. The first part is the first part of the idea episode, counted by reasoning occurrences, rounded down. The same procedure was applied for the last part but using the last part of reasoning occurrences in an idea episode, rounded down. The remaining reasoning occurrences are assigned to the middle part.

Table 4 presents the definitions of the reasoning types used to code for reasoning, derived from the literature review on the three reasoning types. The definitions were chosen to reflect the central characteristics of the three reasoning types, at their core describing the different ways of reaching a conclusion from premises. The interpretation of the coding definitions instructed the coder to code by establishing the 'form' of each utterance. This was necessary to code for the actual way in which each reasoning utterance was presented to the team to avoid coding the implicit meanings behind an utterance instead, which would not be in keeping with the principles of coding verbal reasoning as argued in section 1.2:

a The reasoning utterance reflects the deployment of a mental model that might be different from the ones held by those addressed (Badkeschaub et al., 2007; Johnson-Laird, 1983)

b The verbal enactment of reasoning is influenced by values and beliefs and in turn acts to propose a certain perspective (Dong, 2007; Stumpf \& McDonnell, 2002)

The segment length used here to code for reasoning deviates from other reviewed empirical studies of reasoning (e.g., Dong et al., 2016; Galle, 1996a;

Table 3 Code names and definitions for ideas used in the first step of coding scheme

\begin{tabular}{ll} 
Code name & \multicolumn{1}{c}{ Definition } \\
\hline idea & $\begin{array}{l}\text { Idea coded segments are the un-interrupted sequence of utterances put forward by a } \\
\text { participant proposing an idea. } \\
\text { Idea aspect coded segments are the utterances following idea codes but relating to the } \\
\text { previous idea. Aspects of an idea can be multiple and stated by all participants. Aspects can } \\
\text { also appear after breaks in the sequence of idea-related utterances. }\end{array}$ \\
\hline
\end{tabular}


Table 4 Code names and definitions for reasoning types used in the second step of coding scheme (reference removed for blind review)

\begin{tabular}{ll}
\hline Reasoning code & \multicolumn{1}{c}{ Coding definitions } \\
\hline Abduction & - A hypothesis to account for what is desired or intended (Roozenburg, 1993) \\
& - Creating ideas (to a problem) from imagination (Johnson-Laird, 2009) \\
- A belief held without proof or certain knowledge (Schurz, 2007) & - Preliminary guess to introduce hypotheses (Fann, 1970) \\
& - Definitive and certain conclusion (Schurz, 2007) \\
& - Explicating hypothesis by suggesting consequences (Fann, 1970) \\
- Prediction of result in a given frame (Fann, 1970) & - Proves something must be (March, 1976) \\
& - Explores consequences of an abduction (Fann, 1970) \\
& - Tests a hypothesis with available data (Schurz, 2007) \\
& - Generalises from specific instance or idea (Reichertz, 2010) \\
- Evaluates if something is operative (Fann, 1970) & - Inferring from observed to unobserved (Schurz, 2007) \\
- Induction & Inferring about future courses of events (Johnson-Laird, 2009) \\
\hline
\end{tabular}

Lloyd \& Scott, 1994) as well as reviewed conceptual models (e.g., Dorst, 2011; Roozenburg, 1993) in that the segments (word phrases) do not in themselves contain explicit premises, rules and conclusions. Rather, the coding of the reasoning for such short segments is a consequence of the aim to analyse the reasoning at a micro-level (Rittel, 1987) in which each reasoning segment is dependent on the reasoning pattern (episode) into which it enters. This implies that each micro-level reasoning occurrence can be distinguished and analysed to more accurately describe reasoning patterns.

The coding of reasoning types was restricted to the idea episodes coded in the first step of the coding process because the focus is on the inferences made during the generation of ideas.

Cohen's Kappa was calculated for inter-coder reliability after each of the coding steps (Cohen, 1968). The first and third author coded 460 segments for idea and idea aspect, reaching a Kappa of .71, and 353 segments for reasoning, reaching a Kappa of .61. Both scores are considered 'high' (Fleiss, 1981) or 'substantial' (Landis \& Koch, 1977) and justify the reliability of the coding scheme. The Kappa does not reveal where the specific disagreements come from; however, a qualitative analysis shows that for idea and idea aspect coding, no particular combination of disagreement stands out. For reasoning coding, the primary source of disagreement came from the deduction code, where the second coder tended to code fewer occurrences of the code, while the first coder would code more instances of the code. Since the disagreements occurred in common appearances of multiple deduction codes in a series of uninterrupted segments, the source of error was adapted to refine the code definition for coding the remaining protocols. Disagreements among the coders were discussed, and a common decision was made as to which code to apply. 


\section{Results}

This section presents the results of the analysis. First, the results of the coding provide an overview of the protocol data and the results of the hypotheses. Second, two examples from the protocols illustrate the coding scheme and describe the reasoning patterns found at an individual idea level.

\subsection{Summary of protocol coding results}

The protocols counted 5792 segments of which 2047 (35\%) were idea episodes, i.e., utterances relating to the generation of an idea. Other segments were not determined to concern the generation of ideas in addition to other utterances such as reflection on the design task and social conversation. Idea episodes contained $1698(83 \%)$ reasoning segments, distributed in 218 ideas in total. Table 5 summarises the results.

As observed from the above, deductive reasoning is the most frequent, accounting for $72 \%$ of all reasoning, while abductive reasoning is the second most frequent type of reasoning accounting for $21 \%$.

\subsection{Reasoning patterns in idea episodes}

The hypotheses predict the following development of reasoning patterns in idea episodes.

- H1 predicts a concentration of abductive reasoning in the beginning parts of ideas generated, serving to state an intention or desired result by conjecturing that a specific aspect of the design task is relevant.

- $\mathrm{H} 2$ predicts that deductive reasoning is concentrated in the middle parts of ideas generated, indicating the concretisation of solutions framed by the initial hypothesis.

Figure 1 illustrates the progression of reasoning distribution across the three parts of ideas.

To test the hypotheses, a series of Wilcoxon signed-rank tests are completed. A non-parametric test is applied since the proportional distributions of reasoning in the three idea episode parts are not generally distributed; however, it does show a symmetrical shape in differences among the groups, i.e., the difference of reasoning proportions among each reasoning type across the first,

Table 5 Summary of coding results

\begin{tabular}{llccc}
\hline & & Abductive & Deductive & Inductive \\
\hline Coded reasoning & Count & 349 & 1226 & 123 \\
& Proportion & $21 \%$ & $72 \%$ & $7 \%$ \\
\hline
\end{tabular}


Figure 1 Proportional distribution of reasoning by idea parts with trend lines to emphasise direction

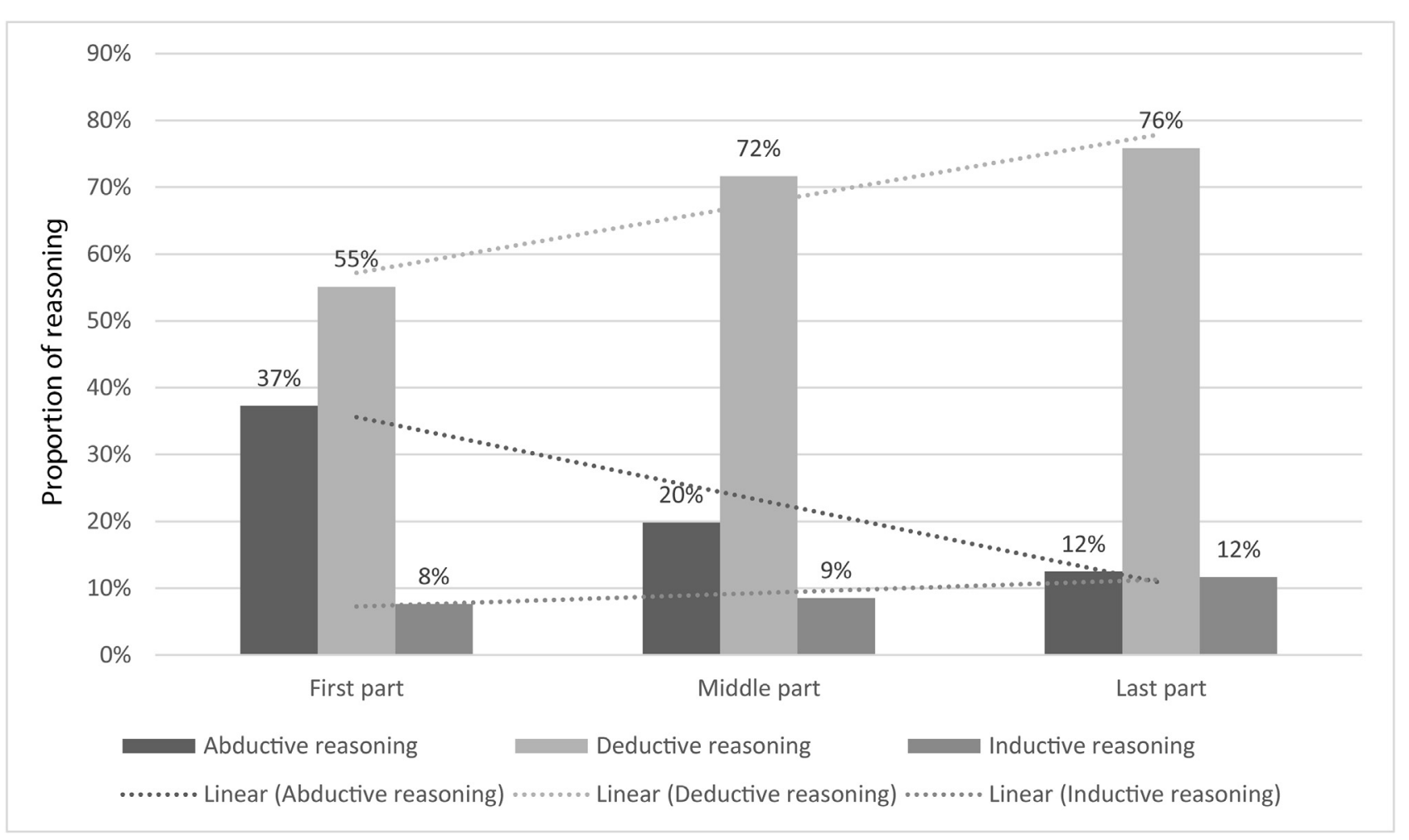


Table 6 Results of Wilcoxon signed-rank tests of hypotheses

\begin{tabular}{lllll}
\hline Reasoning type & \multicolumn{1}{c}{ Tested groups } & $Z$ & $N$ & Significance \\
\hline Abductive & First part, middle part & -5.698 & 203 & $\mathrm{p}<.001$ \\
& First part, last part & -6.716 & 203 & $\mathrm{p}<.001$ \\
Deductive & Middle part, first part & -4.984 & 203 & $\mathrm{p}<.001$ \\
& Middle part, last part & 1.090 & 203 & $\mathrm{p}=.276$ \\
Inductive & Last part, first part & -1.841 & 203 & $\mathrm{p}=.066$ \\
& Last part, middle part & -1.397 & 203 & $\mathrm{p}=.162$ \\
\hline
\end{tabular}

middle and last parts of ideas. Table 6 presents the Wilcoxon signed-rank tests necessary to test hypotheses.

Pertaining to the hypotheses, it was found that:

- Abductive reasoning accounts for $37 \%$ of total reasoning in the first part of ideas and significantly decreases to $20 \%$ in the middle part $(Z=-5.698$, $\mathrm{p}<.001)$ and $12 \%$ in last part $(\mathrm{Z}=-6.716, \mathrm{p}<.001)$, supporting $\mathrm{H} 1$.

- Deductive reasoning accounts for $72 \%$ of total reasoning in the middle part, thus significantly decreasing to $5 \%$ in middle part $(Z=-4.984$, $\mathrm{p}<.001)$, while actually increasing from the middle $(72 \%)$ to the last part $(76 \%)(\mathrm{Z}=1.199, \mathrm{p}=.276)$. This result only supports $\mathrm{H} 2$ from the middle to the first parts, while a direction opposite to what was expected is observed from the middle to the last parts.

As for the exploratory question of the role of inductive reasoning, the results showed that inductive reasoning accounts for $12 \%$ of total reasoning in the last part of ideas, $9 \%$ in the middle part and $8 \%$ in the first part.

Table 6 summarises the test results. Please note that of the 218 total ideas, 203 were of a length that allowed analysing reasoning proportions across all three parts. Aside from the tested hypotheses, the results show that all types of reasoning occur in all parts of ideas and that deductive reasoning is the dominant type across all parts of ideas, accounting for $55 \%, 72 \%$ and $76 \%$ in first, middle and last parts, respectively.

Since the facilitators took part to a limited degree in the idea generation (287 segments coded for reasoning, equivalent to $5 \%$ of all reasoning), the proportional distributions and statistical tests were re-calculated to determine any biases in reasoning patterns caused by the facilitators (despite their being blind to the study's aims and hypotheses). The procedure excluded all complete idea episodes in which a facilitator uttered any reasoning (i.e., segments by facilitators coded for any of the reasoning types), resulting in a reduction of 79 idea episodes. Pertaining to proportional distributions using the reduced data, the results were very similar, showing differences up to $1,5 \%$-points. A re-run of 
the Wilcoxon Signed Rank test confirmed this by showing the same significant and non-significant results as reported in Table 6. As the analysis shows, the participation of facilitators seems not to have interfered with the results.

To further assess the reliability $(\mathrm{R})$ of these results, we conducted a string of analyses aimed at (R1) to assess if the results from the episode split into thirds could be replicated with a mean episode split; (R2) to assess whether the results depend on temporal development within the sessions (e.g., if abductive reasoning happens mainly early in a session) by splitting the transcripts into early/late parts and re-running the analyses; (R3) to test the reliability of the results in each individual team; and (R4) to conduct the same conceptual analyses at a different grain size by looking into the temporal ordering of individual arguments within each episode (as opposed to between as in the main analysis). (R1) All the main results could be replicated with a mean episode split: Abductive reasoning was more prevalent in the first half $(\mathrm{Z}=-5.756$, $\mathrm{p}<.001)$, deductive reasoning was more prevalent in the second half $(\mathrm{Z}=4.147, \mathrm{p}<.001)$, and inductive reasoning showed an increasing, albeit insignificant, trend $(\mathrm{Z}=1.869, \mathrm{p}=.062)$. (R2) Each team's transcript was meansplit into early/late parts. The results indicated that every analysis comparison had the same directionality and approximate size in each split half as they did in the main analysis. Every analysis that was significant in the main result was also significant for each transcript part, and conversely every insignificant main analysis was also insignificant in each transcript part. (R3) All main results were re-run by the team to assess whether the results were driven by a subset of teams. Splitting by team reduces power, and therefore we mainly sought to interpret reliability based on the directionality of the results (as opposed to the significance levels). For abductive reasoning, all five teams replicated a declining effect from both the first to the middle part ( $p$ 's ranging .11 to .002) and from the first to the last parts (p's ranging .078 to .0001). For deductive reasoning, both the increasing effect from the first to the middle part (p's ranging .14 to .0001), and the declining trend from the middle to the last part (p's ranging .91 to .08) were replicable in all five teams. For inductive reasoning, the increasing trend from the first to the last part was found for all five teams, although always insignificant (p's ranging .91 to .06). Less consistent was the inductive increasing trend from the middle to the last part as one team displayed opposite directionality, and one team showed no difference at all. (R4) To assess whether the main results could be replicated at a different grain size, we conducted a reasoning pattern analysis within the episode parts. Given the low count of inductive reasoning, we focused on the interaction between abductive reasoning and deductive reasoning. For each episode part, we counted the number of abductive-deductive (AD) versus deductive-abductive (DA) sequence patterns in terms of the order of which the reasoning types first occurred. The three parts of the episodes differed significantly in their reasoning patterns $\left(\chi^{2}(2)=17.43, \mathrm{p}<.001\right)$. Follow-up two by two chi-square analyses showed that the first part had 
more AD than DA interactions compared to the middle part $\left(\chi^{2}(1)=17.32\right.$, $\mathrm{p}<.001)$; however, they did not differ from the last part $\left(\chi^{2}(1)=2.44\right.$, ns). The middle part conversely differed from the last part, displaying relatively more DA than AD interactions $\left(\chi^{2}(1)=5.11, \mathrm{p}<.03\right)$. These results were replicable with an episode mean split, again showing that the first half of the episodes displayed relatively more AD than DA interactions, compared to the second half $\left(\chi^{2}(1)=13.5 .11, p<.03\right)$. Taken together, the main results appear to be extremely reliable and robust across episode splitting choices, transcript parts, teams, and choice of grainsize. The trending direction was almost uniformly the same in the reliability checks, although the lower $\mathrm{N}$ resulting from splitting the dataset did not always allow for significant results. The overall result is strong support for $\mathrm{H} 1$ and partial support for $\mathrm{H} 2$ and $\mathrm{H} 3$.

To determine whether the groups were internally representative of reasoning in line with the hypotheses, all groups were analysed in relation a) to the overall proportions of reasoning types uttered and b) to whether each group was overly dominated by any single person and whether such persons displayed different reasoning patterns than expected.

Table 7 Idea episode from protocols, translated to English for reporting

\begin{tabular}{|c|c|c|c|c|}
\hline Row & Speaker & Segment & Idea code & $\begin{array}{l}\text { Reasoning } \\
\text { code }\end{array}$ \\
\hline 1 & M & $\begin{array}{l}\text { But maybe you could clean the water sufficiently from one to the } \\
\text { other in a bathroom, }\end{array}$ & idea & abduction \\
\hline 2 & M & so it's not so much about returning it for wastewater treatment & idea & deduction \\
\hline 3 & M & and then all the way back into the infrastructure, & idea & deduction \\
\hline 4 & M & but you take it [the water] from the shower to the toilet, & idea & deduction \\
\hline 5 & M & do a degree that it doesn't create too much foam, & idea & deduction \\
\hline 6 & M & because there are soap leftovers in, or whatever. & idea & deduction \\
\hline 7 & M & If it just fills the toilet cistern... & idea & deduction \\
\hline 8 & M & You could calculate it. & idea & deduction \\
\hline 9 & $\mathrm{~F}$ & Yes, it could be that you could make a closed circuit, & idea aspect & abduction \\
\hline 10 & $\mathrm{~F}$ & for every hotel room, right? & idea aspect & abduction \\
\hline 11 & M & Yes, I like that. & idea aspect & induction \\
\hline 12 & $\mathrm{R}$ & Oh yeah, I mean, then it's your own filth you meet again, right? & idea aspect & induction \\
\hline 13 & $\mathrm{R}$ & You would rather want that, than someone else's. & idea aspect & induction \\
\hline 14 & $\mathrm{~F}$ & Yes & idea aspect & \\
\hline 15 & $\mathrm{R}$ & Yeah, and you could make another closed circuit in the kitchens, & idea aspect & abduction \\
\hline 16 & $\mathrm{R}$ & or the laundry room or the spa. & idea aspect & abduction \\
\hline 17 & M & Then it shouldn't transport so much water at the same time. & idea aspect & deduction \\
\hline 18 & $\mathrm{R}$ & $\begin{array}{l}\text { No, and it can be used, and you can make differentiated } \\
\text { purification methods. }\end{array}$ & idea aspect & deduction \\
\hline 19 & M & Yes & idea aspect & \\
\hline 20 & $\mathrm{R}$ & And possibly you could, if you make the..., I'm don't know how. & idea aspect & \\
\hline 21 & $\mathrm{R}$ & $\begin{array}{l}\text { but then you could go from drinking water to showering water to } \\
\text { kitchen water to cleaning water to toilet water }\end{array}$ & idea aspect & deduction \\
\hline 22 & $\mathrm{R}$ & or whatever it could be so it sort of goes down through, right? & idea aspect & \\
\hline
\end{tabular}


a) Of the 15 participants, $12(80 \%)$ adhered to the same order in terms of the proportions of reasoning. That is, most deductive reasoning was followed by abductive and finally inductive reasoning. Deductive reasoning was the most prevalent for all of the remaining 3 participants.

b) To address the internal distribution in the groups and whether the most active participant would skew the results, our analysis showed that the most active participant in each group contributed with respectively $49 \%, 48 \%, 41 \%, 49 \%$ and $57 \%$ of all group utterances - compared to $33 \%$ if all contributed equally, which is theoretical and not expected. Of these 5 participants, 4 adhered to the overall reasoning ordering (as reported above), while the last person had an equal proportion of abductive and inductive reasoning (both at $18 \%$ ).

Therefore, the reasoning proportion differences at the individual group levels seem not to interfere with the representativity of the overall results.

\subsection{Examples and interpretation of the coding of reasoning}

Two examples are presented to illustrate the reasoning patterns identified from the protocol analyses. This is done by showing coded data supplied with a description of the specific sequences of reasoning occurring.

\subsubsection{Example 1}

Table 7 presents the first example idea episode, including example descriptions of the code definitions used for the three reasoning types. The idea episode begins with an abduction proposing a principle to reuse water (Code definition: 'A hypothesis to account for what is desired or intended', refer to Table 4). Following this, a sequence of deductions occur that argue for why the specific principle is useful by specifying that it is possible to measure the effect of the idea (rows 2-8, Table 7) (Codes: Row 2: 'Prediction of result in a given frame',

Table 8 Idea episode from protocols, translated to English for reporting

\begin{tabular}{lclll}
\hline Row & Speaker & \multicolumn{1}{c}{ Segment } & Idea code & Reasoning code \\
\hline 1 & V & An exterior cover [surrounding the hotel], & idea & deduction \\
2 & V & that can easily be done. & idea & deduction \\
3 & V & But well, it absorbs the dew... & idea & ided \\
4 & V & It's kind of like a membrane within a membrane, okay. & idea & deduction \\
5 & V & So the membrane has these small channels, & idea & deduction \\
6 & V & I mean, it leads the water in these tiny channels, & idea & deduction \\
7 & V & just like the desert rhubarb. & idea & deduction \\
8 & V & Then there are simply these rhubarb leaves forming a surface, & idea & deduction \\
9 & V & then the tiny channels leads the water, & idea & deduction \\
10 & V & and then they [water channels] can lead to some small, local water & idea & deduction \\
& & reservoirs, & idea & deduction \\
11 & V & then it doesn't have to lead it to a large reservoir in the ground, & & \\
\hline
\end{tabular}


rows 3-7: 'Explicating hypothesis by suggesting consequences', row 8: 'Definitive and certain conclusion'). Next, the facilitator $[\mathrm{F}]$ expands the idea by abduction (rows 9-10), building on the initial principle and initiating a new aspect of the idea. This time inductive reasoning follows the abductive reasoning by the remaining team members in the form of an evaluation of the use experience based on personal preference (rows 11-13) (Code: Rows 11-13: 'Inferring about future courses of events'). A team member then proposes to re-contextualise the initial idea principle (rows 15-16) after which deductions determine the effect of the solution (row 17) and a statement (though not explained further) that postulates that an alternative purification method is possible (row 18). Finally, a deduction proposes the possibility of a new principle for reusing water (row 21).

Three immediate observations stand out from the idea episode. First, the example shows that reasoning types occur in a pattern using all three types. Second, concerning the evolution of the idea, all team members partake in the elaboration of the idea through different aspects. Third, there is an interaction between the different occurrences of reasoning, both between and within same reasoning types.

\subsubsection{Example 2}

The second sample idea episode presented in Table 8 is an example of a purely deductive reasoning pattern. The episode begins by proposing an object (an exterior cover) without stating the desired outcome (Table 8, rows 1-2). Thereafter follows the desired outcome, implicitly stated by reference to a solution from the bio-card method (row 3 ). Then, a deductive sequence begins by reusing the structure and principle provided by the bio-card (rows 4-12).

The example shows that reasoning patterns including only deductive reasoning are possible, in this case by analogising from a commonly understood object (the bio-card solution) towards a solution. Additionally, observed from the example is the absence of abductive reasoning and that only one person contributes to the idea.

\section{Discussion}

This section discusses the results from the protocol analyses of reasoning patterns. The first part discusses the observed reasoning patterns by quantitative analysis and by referring to observations made from the patterns of reasoning found in specific idea episodes. The second part proposes three arguments from the basis of the study before discussing the implications of the results and proposes avenues of future research. 


\subsection{Hypotheses and general reasoning patterns}

As the results of $\mathrm{H} 1$ show, there is a significant concentration of abductive reasoning in the first parts of ideas. Hence, the finding is consistent with the reviewed models of design activity that assume an abductive stage to initiate instances of inference-making in design activity (March, 1976; Schön, 1991). $\mathrm{H} 2$ was only partially supported, finding deductive reasoning to increase as an idea progresses. The surprising prevalence of deductive reasoning persists throughout all idea parts $(55-76 \%)$, while there is a significant concentration of abductive reasoning in the first parts. Hence, there is support for a theoretically proposed two-stage process involving abductive-deductive patterns, with only a few occurrences of inductive reasoning and all the while dominated by deductive reasoning across all parts of ideas. The results showed that the exploratory question pertaining to the presence of inductive reasoning can be negatively answered in the sense that inductive reasoning is the least prevalent type of reasoning. Rather, the last parts of ideas were the most concentrated parts of deductive reasoning.

The tests to determine any differences between groups with or without fully experienced participants, as well as across various robustness checks, did not show any variations of the reasoning patterns. Hence, we observe that the reasoning patterns across many different factors, including the experience of the participants, temporal placement in idea generation sessions and more, are very robust and follow abductive-deductive patterns.

The following section discusses the coded reasoning types and patterns using specific occurrences from the above presented examples.

Abduction-coded segments tend to occur in an uncertain form that at the same time proposes new frames or perspectives on functions to achieve to address the design task of saving water. The frames are not absolute and are observed to change as the idea progresses through re-framings that proposes new problem settings and aspects of ideas (Dorst \& Cross, 2001; Schön, 1991). Concretely, example 1 shows how a first abduction is made (Table 7, row 1) in which a specific perspective that can possibly lead to the saving of water is introduced. Later, in the same example, abductions occur again to reframe the initial perspective (rows 9-10) and again later introduced a subfunction to the previous (rows 15-16).

Deduction-coded segments function to derive effects in response to the frames and appear as causal inference chains that lead to a conclusion. Often, these deductions draw on prior abductive reasoning as the premise. Further, they do not produce guaranteed objective 'truths'. Rather, the deductions serve to explore and concretise the framing to amend and discern the validity of the abduction (Johnson-Laird, 2009; Schön, 1991). The two examples each 
provide an explanation to the high proportion of deductive reasoning found in the protocols. Example 1 shows that deductive reasoning can occur as a series of deductions functioning to describe a solution (Table 7, rows 2-8) to a prior abduction (row 1), which is similar to the notion of mental simulation (only in a verbal form), found to be a strategy for resolving uncertainty in design activity (Christensen \& Schunn, 2009). Example 2 shows a different deductive reasoning sequence (Table 8) that involves the analogical mapping of a solution provided by the bio-card design method to the design task at hand. This can be interpreted as an instance of direct analogical transfer (Ahmed \& Christensen, 2009), a strategy found to be mostly used by novice designers (ibid.). However, the example also holds similarity to the notions of explanatory abductive reasoning (Roozenburg, 1993) and abduction-1 type reasoning (Dorst, 2011) in that the reasoning pattern (here coded as deductive) follows an implicit abductive explanation that uses a known solution in a new context resulting in a causal explanation. The difference in ways of perceiving reasoning among the conceptual models of reasoning in design and the present verbal reasoning approach is discussed in greater detail in section 5.2.

Induction-coded segments are shown in example 1 to occur in the form of the informal appraisal of an idea, in the example as personal preference (Table 7 , row 11), or as a combination of evaluating the consequence of an idea and personal preference (rows 12-13). However, these are not instances of reasoning suitable for evaluation in relation to the discussed models of design and the formal role of inductive reasoning as the generalisation of the specific to the general (Peirce, 1980). Rather, the empirical analysis of inductive reasoning implies for inductive reasoning that the expression of personal preference (e.g., the utterance 'Yes, I like that' found in row 11, Example 1) as part of the verbal form of some underlying acceptance of what was previously proposed. This acceptance is based on some previous knowledge or experience or even attitude towards a specific idea. However, since the reasoning is argumentative, it holds a possible importance to the dialogue as it promotes a positive attitude and agreement that might spur the continuation of other members of a group.

However, despite the negligible role of inductive reasoning, we contend that the observed abductive-deductive patterns (discussed at length in section 5.2) in part makes up for the lack of evaluation through mental simulation (Christensen \& Schunn, 2009). That is, for deductive reasoning, the utterances put forward (often in sequences) that are assumed to 'explicate' and explore an insight from the premise provided one possible consequence of a premise (or frame). Thus, this one possible solution is not without importance since it would continue to be one of the more relevant solutions to a proposed frame (assuming that uttered ideas are better than ideas never put forward by anyone in a team). In turn, such solution would then satisfy the need for exploring a given frame, thus simulating and evaluating an idea. Indeed, similar studies of 
design reasoning have found deductive reasoning to be evaluative (Dong et al., 2015) in relation to ideas.

\subsection{Verbal reasoning in idea generation processes}

Drawing from the results of the tested hypotheses and the observations made from the ideas episodes, we make three primary arguments and discuss their implications and the need for future research. The first argument is that abductive-deductive sequences are a central component of micro-level design activity. The second is that empirically analysing reasoning necessitates perceiving reasoning patterns unlike those proposed in conceptual proposals of reasoning in design. The third is that verbal reasoning is indicative of the mental models held by individuals. In the following section, the implications of the study's results for design practice are discussed.

\subsubsection{Abductive-deductive patterns dominate design idea generation}

First, pertaining to the reasoning pattern observed from the analysis of the proportional distribution, and tested by the hypotheses, we argue that an abductive-deductive pattern is appropriate for describing design activity in a context of idea generation, which is similar to what Roozenburg and Cross (1991) describe as analysis-synthesis cycles, or as the operation between concept and knowledge domains, as proposed by $\mathrm{C}-\mathrm{K}$ theory (Hatchuel \& Weil, 2008). As such, the reasoning types enter into patterns of inference that interact among abductive reasoning, found to be significantly concentrated at the beginning of ideas, and deductive reasoning is concentrated in the later parts of ideas but dominant throughout. Abductive reasoning proposes frames or perspectives for addressing the main design task, while deductive reasoning in turn explores how such a frame is viable to actually address the design task through simulation and thus allows determining the validity of solutions (Johnson-Laird, 2009; Lloyd \& Scott, 1994). This process then repeats or iterates, resulting in variations of the original frames, as exemplified by Table 7, rows 1-10. As such, we contend that the abductive-deductive cycles of reasoning without any explicit inductive reasoning to evaluate are not indicative of aimless activity. Rather, the cycles show similarity to what several models of design propose as a core tenet to design - the ability to quickly iterate among phases that are divergent and convergent, whether defined as mental simulation (Christensen \& Schunn, 2009), generative sensing (Dong et al., 2016) or composition and decomposition (March, 1976). Aside from showing the presence of such patterns empirically, the present study contributes by showing how different reasoning types interact and are interdependent, as further discussed below.

As the empirical data analysed in the present study focuses on idea generation activity, the future research should focus on analysing reasoning patterns in 
similar ways during other stages of design activity, e.g., concept development or detail design, to ascertain whether the same disorderly and opportunistic behaviour are present, and whether the abductive-deductive pattern is still a pronounced component of design activity. Work exists in this regard (see Ball, Evans, Dennis, \& Ormerod, 1997; Visser, 1994) but draws conclusions based on analysing design activity in segments larger than treated here and as such does not allow for analysis of how inferences are made at a microlevel. Further, the research in this avenue is limited to instances of design activity whereby group design and argumentation among people naturally occurs to satisfy the presently applied methodological approach.

\subsubsection{Micro-level design activity contains interactions between reasoning types}

Second, as the results show, the applied coding scheme ascertains reasoning patterns at a micro-level of design activity and allows describing the processes involved in generating ideas in great detail. While a prominent abductivedeductive pattern exists, the presented examples of idea episodes show how reasoning types occur in chains of reasoning in different types and in disorderly patterns that do not necessarily adhere to formal reasoning types. These interactions among reasoning types further show that each individual instance of reasoning (at the coded micro-level) is interdependent to the other instances in which it is put forward - regardless of whether it is from the same person. Hence, a micro-level analysis of reasoning such as this then improves the understanding of design activity (Rittel, 1987) by showing the how different reasoning types interact by drawing conclusions using different patterns of inference, for example, the use of deductive reasoning to arrive at a solution under a given framing or the use of inductive reasoning to evaluate a framing with no presence of deductive reasoning. Such observations imply that design activity does not follow a strictly logical form but is also informal and comprises un-structured and opportunistic activities (Ball \& Ormerod, 1995). As also shown, these activities are shared, showing a resemblance to the concept of shared cognition (DeChurch \& Mesmer-Magnus, 2010) or team mental models (Dong, Kleinsmann, \& Deken, 2013), which are indicators of the ability of groups to successfully work together. Further research is necessary in this direction to better understand how different types of reasoning behaviour, e.g., using different patterns, influences the solutions generated. One possible approach to this is the use of linkography (Goldschmidt, 2014) or similar means to identify influences among ideas and to determine the quality of generated ideas (Kudrowitz \& Wallace, 2012).

The analysis method applied in the research is based on the proposal that micro-level, argumentative, reasoning is key to understand design activity (Rittel, 1987). While other reviewed studies of design reasoning apply different methodologies, interpretations of reasoning and/or unit of analysis (e.g., Dong 
et al., 2015; Galle, 2002; Lloyd \& Scott, 1994), we do not presume our method to replace such methods. Rather, our approach allows a different perspective on reasoning in idea generation specifically. The micro-level perspective on design reasoning offered here has the advantage of capturing reasoning as it is actually put forward to other members in a design team; however, it is limited in that it does not capture implicit, or taken for granted, common understandings in a team. The study thus offers an alternative interpretation of reasoning in design activity that is in contrast to existing conceptual models of design reasoning (Dorst, 2011; March, 1976; Roozenburg, 1993)as well as other empirical studies of reasoning (Galle, 1996a; Lloyd \& Scott, 1994).

\subsubsection{Verbal reasoning is argumentative}

Third, acknowledging that verbal reasoning is influenced by values and intentions (Roozenburg, 1993; Stumpf \& McDonnell, 2002) and that verbal reasoning is a process of argumentation (Rittel, 1987) that enacts design and thus influences design activity (Dong, 2007), we argue that the reasoning analysed here is inherently subjective and non-monotonous (McDonnell, 2012). This implies that while one participant in group design activity may use deductive reasoning because an inference fits a held mental model, the same inference may not be 'true' and thus is not suitable for deductive inference, in the group mental model, or with another participant. Therefore, the reasoning used during group design activity has a dual function of both making inferences towards the generation of new ideas and also being indicative of the mental models held by the members of that group, regardless of whether they are shared by other group members. Hence, reasoning is argumentative, underscoring the importance of the performative aspect of verbal reasoning when empirically analysing design activity. One approach to further investigate the argumentative aspect of design reasoning is the analysis of the mental models held among the members of design teams in correlation with identified reasoning patterns (Badke-schaub et al., 2007). Such an analysis could provide insights into how coordinated or shared a group's understanding of a design task is, which is shown to influence performance (ibid.).

\subsubsection{Implications for design practice}

For design practice, the results of the study provide a basis for understanding the cognitive processes involved in group idea generation and has at least two implications. First, the results can be used to develop AI tools to monitor and diagnose the dialogue among groups engaged in design activity and to make suggestions for changes to behaviour to intervene if, e.g., detected reasoning sequences do not adhere to abductive-deductive patterns. Second, the study has implications for the development of new, creative methods for idea generation as such methods should support the abovementioned abductivedeductive patterns. In particular, as the study finds empirical evidence for the centrality of abductive-deductive patterns in all part of ideas in the early 
stages of idea generation, the implications are that such creativity methods should support and allow deductive reasoning to be made in a dynamic and productive interaction with abductive reasoning rather than promoting division, meaning that abductive reasoning comes first, only allowing deductive reasoning at later stages (i.e., by only focusing on new idea perspective at first without allowing the utilisation of group resources to explore those ideas until later stages). Hence, the abductive-deductive dynamic can be seen as similar to hermeneutic circles or experiential learning allowing groups of designers to learn about how to generate solutions and re-interpret design tasks even at the micro-level of early stage design activity.

Finally, the development of methods or tools to support reasoning in design practice must allow for the inherently argumentative characteristic of design. Hence, such methods should focus on making designers explicate their beliefs and test their assumptions. This could, for example, be done through the development of automated tools to monitor and diagnose design activity in relation to espoused reasoning patterns. Such tools could potentially support and intervene in design activity in situ.

\subsection{LIMITATIONS}

The present study of reasoning in design draws from empirical data from idea generation activity. Hence, the implications primarily concern such idea generation activity. However, as the hypotheses draw on models of design activity in general, the study allows for observations to be made on design activity in general. This is primarily done in the explanation and discussion of the examples in the study. To further qualify these observations, the paper also relates them to other behaviours of importance to design activity such as the use of mental simulations (B.T. Christensen \& Schunn, 2009) or analysis-synthesis (Roozenburg, 1993). To address the generalisability of the research, further research is necessary at other stages of design activity.

Further, the study's design limits the making of observations or conducting analyses as to whether certain patterns or characteristics of verbal reasoning lead to greater value or effectiveness in idea generation activity than others. Future studies should analyse reasoning patterns to reliable outcome measures to idea 'quality', which in turn would make it possible to develop methods or tools that promote such beneficial reasoning behaviour.

There is a relatively low number of participants in the study (15 participants divided into five teams). However, as argued above, the level of segmentation of the protocols becomes unmanageable if it is much larger. A possible remedy is to code for reasoning in larger segments, which has already been done elsewhere (see Galle, 1996b; Lloyd \& Scott, 1994). 
The limited participation of the facilitators in the idea generation could potentially affect the results. To account for this, analyses of the data excluding idea episodes in which the facilitators contributed to reasoning were completed and showed no significant differences and the same directionality in the results.

To capture the different reasoning types, the coding scheme is dependent on reasoning made verbally explicit to ascertain reasoning patterns. Hence, the study captures reasoning as it is put forward in an argumentative form (Mercier \& Sperber, 2011; Perelman \& Olbrechts-Tyteca, 1973) in a group setting without instructional priming to think-aloud, which, we argue, is representative of the actual verbal reasoning taking place within groups of people in design activity (Rittel, 1987). Future research might attempt to instruct participants to clarify all their arguments to assess whether any individual covert reasoning arguments may be made explicit upon instruction.

\section{Conclusion}

An empirical study of the reasoning types used in group idea generation for a design task was conducted with participants from industry. Five teams were recorded, and the transcribed protocols were analysed using a coding scheme for determining the presence of ideas and reasoning types in verbal data in more than 5500 segments. The study aimed to empirically analyse verbal reasoning patterns through the analysis of protocol data.

The results suggest that reasoning in design activity across hundreds of ideas follow a general pattern of abductive-deductive reasoning. The study found that abductive reasoning is concentrated in the first part of ideas, functioning to frame and propose solutions. Deductive reasoning, concentrated in the latter two parts of ideas, was the most frequent across all idea parts, functioning to explore and concretise the solutions proposed by the initial, often abductive, framing. Inductive reasoning, the least frequent of the reasoning types, did not appear to have a pronounced effect in idea generation activity. At the individual idea level, reasoning patterns were found to be disorderly but to contain elements of abductive-deductive patterns.

The study found that reasoning instances are understood as chains of inference that accumulate and create understanding of approaches to address overall problem settings in design, indicating that the analysis of reasoning patterns at a micro-level of design activity holds potential to advance the understanding of design activity and be applied to develop support tools and methods given future research.

This study contributes to an understanding of design activity at a micro-level in a real-world setting. From the protocol analysis of design teams, patterns of verbal reasoning during idea episodes were identified. The study is novel in 
that it proposes an approach to analyse reasoning patterns at the micro-level of design activity, allowing for the identification of central abductivedeductive patterns of reasoning from empirical data, paving the way for several strands of future research.

\section{Acknowledgment}

The authors acknowledge the support of the industry practitioners participating in the study and the graduate students acting as facilitators. We appreciate the comprehensive and constructive feedback from the reviewers helping to make the paper ready for publication. This research did not receive any specific grant from funding agencies in the public, commercial, or non-for-profit sectors.

\section{References}

Ahmed, S., \& Christensen, B. T. (2009). An in situ study of analogical reasoning in novice and experienced design engineers. Journal of Mechanical Design, 131(11). 111004-1-9.

Ahmed, S., Wallace, K., \& Blessing, L. (2003). Understanding the differences between how novice and experienced designers approach design tasks. Research in Engineering Design, 14, 1-11.

AskNature. (n.d.). AskNature bio-mimicry taxonomy. Retrieved January 10, 2016, from http://www.asknature.org/article/view/biomimicry_taxonomy

Badke-schaub, P., Lauche, K., Neumann, A., \& Ahmed, S. (2007a). Task - team - process: Assessment and analysis of the development of shared representations in an engineering team. In J. McDonnell, \& P. LLoyd (Eds.), About: Designing. Analysing design meetings. Leiden, The Netherlands: CRC Press/ Balkema.

Badke-Schaub, P., Neumann, A., Lauche, K., \& Mohammed, S. (2007b). Mental models in design teams: A valid approach to performance in design collaboration? CoDesign, 3(1), 5-20.

Ball, L. J., Evans, J. S. B. T., Dennis, I., \& Ormerod, T. C. (1997). Problem-solving strategies and expertise in engineering design. Thinking \& Reasoning, 3(4), $247-270$.

Ball, L., \& Ormerod, T. (1995). Structured and opportunistic processing in design: A critical discussion. International Journal of Human-Computer Studies(43), $131-151$.

Bucciarelli, L. L. (2002). Between thought and object in engineering design. Design Studies, 23(3), 219-231.

Chi, M. T. H. (1997). Quantifying qualitative analyses of verbal Data : A practical guide. The Journal of the Learning Sciences, 6(3), 271-315.

Christensen, B. T. (2010). Images of users and products shown during design processes increase users willingness to use the design outcome. Artificial Intelligence for Engineering Design, Analysis and Manufacturing: AIEDAM, 24(2), 179-189.

Christensen, B. T., \& Ball, L. J. (2014). Studying design cognition in the real world using the "in vivo" methodology. In P. Rodgers, \& J. Yee (Eds.), The routledge companion to design research (pp. 317-328). Abingdon, UK: Routledge.

Christensen, B. T., \& Schunn, C. D. (2009). The role and impact of mental simulation in design. Applied Cognitive Psychology, 23(3), 327-344. 
Cohen, J. (1968). Weighted Kappa: Nominal scale agreement with provision for scaled disagreement or partial credit. Psychological Bulletin, 70(4), 213-220.

Cross, N. (2001). Design cognition: Results from protocol and other empirical studies of design activity. In C. Eastman, W. Newstetter, \& M. McCracken (Eds.), Design knowing and learning: Cognition in design education (pp. 79-103). Oxford: Elsevier.

de Bono, E. (1992). Serious creativity: Using the power of lateral thinking to create new ideas. Michigan: HarperBusiness.

DeChurch, L. a, \& Mesmer-Magnus, J. R. (2010). The cognitive underpinnings of effective teamwork: A meta-analysis. Journal of Applied Psychology, 95(1), $32-53$.

Dong, A. (2007). The enactment of design through language. Design Studies, 28(1), 5-21.

Dong, A., Garbuio, M., \& Lovallo, D. (2016). Generative sensing in design evaluation. Design Studies, 45, 68-91.

Dong, A., Kleinsmann, M. S., \& Deken, F. (2013). Investigating design cognition in the construction and enactment of team mental models. Design Studies, 34(1), 1-33.

Dong, A., Lovallo, D., \& Mounarath, R. (2015). The effect of abductive reasoning on concept selection decisions. Design Studies, 37, 37-58.

Dorst, K. (2011). The core of 'design thinking' and its application. Design Studies, $32(6), 521-532$.

Dorst, K., \& Cross, N. (2001). Creativity in the design process: Co-evolution of problem-solution. Design Studies, 22, 425-437.

Ericsson, K. A., \& Simon, H. A. (1993). Protocol analysis (revised ed). Cambridge, MA: MIT Press.

Fann, K. T. (1970). Peirce's theory of abduction. The Hague: Martinushoff.

Fleiss, J. L. (1981). Statistical methods for rates and proportions (2nd ed.). New York: John Wiley.

Galle, P. (1996a). Design rationalization and the logic of design: A case study. Design Studies, 17(3), 253-275.

Galle, P. (1996b). Replication protocol analysis: A method for the study of realworld design thinking. Design Studies, 17, 181-200.

Galle, P. (2002). Exploring implicit design knowledge by logical analysis of arguments. In P. Galle, \& G. E. Laster (Eds.), Knowledge for creative decisionmaking: Proceedings of a special focus symposium under InterSymp-2002 in baden-baden, Germany (pp. 27-31). Windsor: International Institute for Advanced Studies in Systems Research and Cybernetics.

Goldschmidt, G. (2009). The dialects of sketching. Creativity Research Journal, $4(2), 123-143$.

Goldschmidt, G. (2014). Linkography: Unfolding the design process. Cambridge, MA: MIT Press.

Hatchuel, A., \& Weil, B. (2008). C-K design theory: An advanced formulation. Research in Engineering Design, 19(4), 181-192.

Johnson-Laird, P. N. (1983). Mental models. Cambridge: Cambridge University Press.

Johnson-Laird, P. N. (2009). How we reason. Oxford: Oxford University Press.

Klimoski, R., \& Mohammed, S. (1994). Team mental model: Construct or metaphor? Journal of Management, 20(2), 403-437.

Koskela, L., Paavola, S., \& Kroll, E. (2018). The role of abduction in production of new ideas in design. In P. E. Vermaas, \& S. Vial (Eds.), Advancements in the philosophy of design (pp. 153-183). Springer. 
Kroll, E., \& Koskela, L. (2015). On abduction in design. In J. S. Gero (Ed.), Design computing and cognition '14 (pp. 327-344). London: Springer.

Kudrowitz, B. M., \& Wallace, D. (2012). Assessing the quality of ideas from prolific, early-stage product ideation. Journal of Engineering Design, 24(2), 1-20.

Landis, R. J., \& Koch, G. G. (1977). The measurement of observer agreement for categorical data. Biometrics, 33(1), 159-174.

Lloyd, P., \& Scott, P. (1994). Discovering the design problem. Design Studies, 16, $125-140$.

Magnani, L. (1995). Creative processes in scientific discovery. European Journal for High Ability, 6(2), 160-169.

Magnani, L. (2004). Reasoning through doing. Epistemic mediators in scientific discovery. Journal of Applied Logic, 2(4), 439-450.

March, L. (1976). The logic of design. In L. March (Ed.), The architecture of form (pp. 16-40). Cambridge: Cambridge University Press.

McDonnell, J. (2012). Accommodating disagreement: A study of effective design collaboration. Design Studies, 33(1), 44-63.

Mercier, H., \& Sperber, D. (2011). Why do humans reason? Arguments for an argumentative theory. Behavioral and Brain Sciences, 34(2), 57-74, discussion 74-111.

Merriam-Webster. (n.d.). Idea definition. Retrieved December 3, 2015, from http://www.merriam-webster.com/dictionary/idea

Osborn, A. F. (1953). Applied imagination. Oxford, England: Scribner.

Oxford. (n.d.). Idea definition. Retrieved December 3, 2015, from http://www.oxforddictionaries.com/definition/english/idea

Peirce, C. S. (1980). The collected papers of charles sanders Peirce. Cambridge, MA: Belknap Press.

Perelman, C., \& Olbrechts-Tyteca, L. (1973). The new rhetoric: A treatise on argumentation. Notre Dame: Notre Dame Press.

Polk, T. a., \& Newell, A. (1995). Deduction as verbal reasoning. Psychological Review, 102(3), 533-566.

Reichertz, J. (2010). Abduction: The logic of discovery of grounded theory. Forum for Qualitative Social Research, 11(1).

Rittel, H. W. J. (1987). The reasoning of designers. Delivered at the international congress on planning and design theory. Boston.

Roozenburg, N. F. M. (1993). On the pattern of reasoning in innovative design. Design Studies, 14(1), 4-18.

Roozenburg, N. F. M. (1995). What is Design? In N. F. M. Roozenburg (Ed.), Product design: Fundamentals and methods (pp. 53-81) New York: John Wiley and Sons.

Roozenburg, N. F. M., \& Cross, N. G. (1991). Models of the design process: Integrating across the disciplines. Design Studies, 12(4), 215-220.

Schön, D. A. (1991). The reflective practitioner: How professionals think in action. London: Routledge.

Schurz, G. (2007). Patterns of abduction. Synthese, 164(2), 201-234.

Stumpf, S. C., \& McDonnell, J. T. (2002). Talking about team framing: Using argumentation to analyse and support experiential learning in early design episodes. Design Studies, 23(1), 5-23.

Valkenburg, R., \& Dorst, K. (1998). The reflective practice of design teams. Design Studies, 19(3), 249-271.

Visser, W. (1994). Organisation of design activities: Opportunistic, with hierarchical episodes. Interacting with Computers, 6(3), 239-274.

Voss, J. F. (2006). Toulmin's model and the solving of ill-structured problems. Argumentation, 19(3), 321-329. 